INPLASY PROTOCOL

To cite: Li et al. Effects of DRP1 on tumor metastasis: a systematic review. Inplasy protocol 202040208. doi: 10.37766/inplasy2020.4.0208

Received: 29 April 2020

Published: 30 April 2020

Corresponding author: Xiaohu Wang

xhwanggansu@163.com

Author Affiliation:

The First School of Clinical Medicine, Lanzhou Uni

Support: 2017-RC-23

Review Stage at time of this submission: Preliminary searches.

Conflicts of interest: No potential conflicts of interest were disclosed.

\section{Effects of DRP1 on tumor metastasis: a systematic review}

Li, CC1; Zhang, QN2; Li, Z3; Feng, SW4; Geng, YC5; Wang, LN6; Zhao, XS7; Yang, $\mathrm{KH}^{8}$; Liu, Y9; Wang, $\mathrm{XH}^{10}$.

Review question / Objective: To investigate the correlation between DRP1 and tumor metastasis.

Condition being studied: Tumor metastasis to vital organs is the most important biological behavior in cancer patients. It is responsible for a significant obstacle to treatment and is the main cause of most cancer-associated mortality. The high expression of DRP1 is found in cancers such as rectal cancer, oncocytic thyroid tumors and melanoma. In a recent study by Huang et al., they demonstrated that patients with high phospho-DRP1Ser616 showed high risk on poor disease-free survival $(P=0.0003)$ and 5-year OS $(P=0.002)$ in locally advanced rectal cancer. What's more, phospho-DRP1Ser616 contributes to metastasis and treatment resistance. Literature data also documented that DRP1 positivity was in $65 \%$ and $90 \%$ of advanced cutaneous melanoma and oral melanoma cases. In addition, DRP1 expressions were related to advanced Clark's levels in cutaneous melanoma. These outcomes suggested that DRP1 up-regulation might be an early event in some types of cancers, and also support a crosstalk between mitochondrial fragmentation and tumor growth and metastasis.

INPLASY registration number: This protocol was registered with the International Platform of Registered Systematic Review and Meta-Analysis Protocols (INPLASY) on 30 April 2020 and was last updated on 30 April 2020 (registration number INPLASY202040208).

\section{INTRODUCTION}

Review question / Objective: To investigate the correlation between DRP1 and tumor metastasis.

Condition being studied: Tumor metastasis to vital organs is the most important biological behavior in cancer patients. It is responsible for a significant obstacle to treatment and is the main cause of most cancer-associated mortality. The high expression of DRP1 is found in cancers such as rectal cancer, oncocytic thyroid tumors and melanoma. In a recent study by Huang et al., they demonstrated that patients with high phospho-DRP1Ser616 showed high risk on poor disease-free 
survival $(P=0.0003)$ and 5-year OS $(P=$ 0.002 ) in locally advanced rectal cancer. What's more, phospho-DRP1Ser616 contributes to metastasis and treatment resistance. Literature data also documented that DRP1 positivity was in $65 \%$ and $90 \%$ of advanced cutaneous melanoma and oral melanoma cases. In addition, DRP1 expressions were related to advanced Clark's levels in cutaneous melanoma. These outcomes suggested that DRP1 up-regulation might be an early event in some types of cancers, and also support a crosstalk between mitochondrial fragmentation and tumor growth and metastasis.

Rationale: Given the important role of DRP1 in the metastatic cascade, a molecular understanding of how DRP1 controlled in tumor cells migrating is of critical importance. In this context, it would be interesting to investigate the correlation between DRP1 and tumor metastasis.

\section{METHODS}

Search strategy: terms: "Dynamin-related protein 1", "DRP1", "DNM1L", "Metastasis" and "Metastases" databases: pubmed, web of science, ovid, embase, chinese biomedical database and cochrane library.

Participant or population: Patients, animals or cell lines.

Intervention: The key to regulate the dynamic changes of mitochondrial morphology are so-called "mitochondriashaping" proteins. The dynamin-related protein 1 (DRP1) is one of the most related proteins that initiate mitochondrial fission by forming loops and loops. DRP1 is a cytosolic protein, then phosphorylation at Ser616 or dephosphorylation at Ser637 promote DRP1 translocate to mitochondria and induce mitochondrial division. The high expression of DRP1 is found in cancers such as rectal cancer, oncocytic thyroid tumors and melanoma. DRP1 up-regulation might be an early event in some types of cancers, and also support a crosstalk between mitochondrial fragmentation and tumor growth and metastasis.
Comparator: Normal tissue, adjacent normal tissue or normal cells.

Study designs to be included: No study designs limited.

Eligibility criteria: Studies that quantitatively or semi-quantitatively evaluated the expression of DRP1 and explored the relevant pathogenesis between DRP1 and tumor metastasis were considered. In vivo and in vitro studies were included, as were animal models and human trials.

Information sources: Databases (PubMed, Web of science, Embase, OVID, Chinese Biomedical Database and Cochrane library); contact with authors; trial registers; references.

Main outcome(s): The primary outcome was that DRP1 regulated the migration and invasion of cancer cells.

Additional outcome(s): The second outcome was that the mechanism of DRP1 regulated the motility of cancer cells.

Data management: Firstly, deletion duplicate records; secondly, removing publications with inclusion criteriaineligible after reading titles and abstracts; finally, clear included papers after reading full texts.

Quality assessment / Risk of bias analysis: Not applicable.

Strategy of data synthesis: We will provide a narrative synthesis of the findings from the included studies.

Subgroup analysis: This is a qualitative synthesis; subgroup analysis is not planned.

Sensibility analysis: Not applicable.

Language: English.

Country(ies) involved: China. 
Keywords: DRP1; metastasis; systematic review.

Contributions of each author:

Author 1 - Chengcheng Li - write the manuscript.

Author 2 - Qiuning Zhang - choose research directions.

Author 3 - Zheng Li - guide the writing practice.

Author 4 - Shuangwu Feng - screened literatures.

Author 5 - Yichao Geng - screened literatures.

Author 6 - Lina Wang - search publications. Author 7 - Xueshan Zhao - search publications.

Author 8 - Kehu Yang - guide the writing practice.

Author 9 - Yang Liu - decisions maker.

Author 10 - Xiaohu Wang - decisions maker. 